\title{
EResearch Square \\ CRISPR-Assisted Detection of RNA-Protein Interactions in Living Cells
}

\section{Wenkai Yi}

Department of Biomedical Sciences, City University of Hong Kong, Hong Kong SAR, China Jingyu Li

Department of Biomedical Sciences, City University of Hong Kong, Hong Kong SAR, China

\section{Xiaoxuan Zhu}

Department of Biomedical Sciences, City University of Hong Kong, Hong Kong SAR, China

\section{Xi Wang}

School of Medicine, Northwest University, Xi'an, China

\section{Ligang Fan}

School of Medicine, Northwest University, Xi'an, China

\section{Wenju Sun}

School of Medicine, Northwest University, Xi'an, China

\section{Linbu Liao}

School of Medicine, Northwest University, Xi'an, China

\section{Jilin Zhang}

Department of Medical Biochemistry and Biophysics, Karolinska Institutet, Stockholm, Sweden

\section{Xiaoyu Li}

School of Medicine, Northwest University, Xi'an, China

\section{Jing Ye}

Department of Pathology, The Fourth Military Medical University, Xi'an, China

\section{Fulin Chen}

School of Medicine, Northwest University, Xi'an, China

\section{Jussi Taipale}

Department of Medical Biochemistry and Biophysics, Karolinska Institutet, Stockholm, Sweden

Kui Ming Chan ( $\nabla$ ming.chan@cityu.edu.hk)

Department of Biomedical Sciences, City University of Hong Kong, Hong Kong SAR, China

\section{Liang Zhang ( $\sim$ liangzhang.28@cityu.edu.hk)}

Department of Biomedical Sciences, City University of Hong Kong, Hong Kong SAR, China

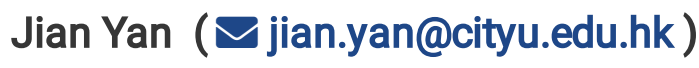

Department of Biomedical Sciences, City University of Hong Kong, Hong Kong SAR, China

\section{Method Article}


Keywords: IncRNA, RNA binding protein, CRISPR/CasRx, BASU, Mass spectrometry

Posted Date: June 22nd, 2020

DOl: https://doi.org/10.21203/rs.3.pex-904/v1

License: (c) (1) This work is licensed under a Creative Commons Attribution 4.0 International License. Read Full License

Version of Record: A version of this preprint was published at Nature Methods on June 22nd, 2020. See the published version at https://doi.org/10.1038/s41592-020-0866-0. 


\section{Abstract}

Delineating the protein network associated with long non-coding RNAs (IncRNAs) is fundamental to understanding the functional mechanisms of IncRNAs. Current methods to identify IncRNA-binding proteins either rely on crosslinking-mediated complex co-precipitation or require extensive molecular engineering, leading to drawbacks such as loss of cellular context and low capture efficiency. Here we describe a CRISPR-Assisted RNA-Protein Interaction Detection method (CARPID), which leverages CRISPR/CasRx-based RNA targeting and proximity labeling, to rapidly capture binding proteins of specific IncRNAs in their native cellular context followed by LC-MS/MS identification. Applied to a variety of IncRNAs of different lengths and subcellular localizations, CARPID is proven to be a reliable and robust tool to discover the binding proteins of IncRNAs inside living cells.

\section{Introduction}

Although only $2 \%$ of the human genome codes for protein sequences ${ }^{1}$, more than $70 \%$ of the genomic DNA can be transcribed to RNA at various stages during development ${ }^{2}$. For decades, these tremendous numbers of non-coding RNAs (ncRNAs) were recognized as "dark matter" and their function remains underexplored. Recent interests have been raised upon these ncRNAs, especially the long non-coding RNAs (IncRNA, defined as ncRNA of more than 200 nucleotides in length) that become widely accepted as important components participating in epigenetic regulation. Identification of IncRNA associated proteins will reveal the molecular mechanism of its cellular function in complex human disorders.

Despite the growing appreciation of the functional importance of RNA binding proteins (RBPs), technical limitations exist in elucidating IncRNA-protein interactions in living cells. The current methods mostly depend upon chemical or UV-mediated crosslinking between RNA and protein molecules for efficient complex isolation ${ }^{3-6}$. Such procedures may generate non-systematic bias and mask the interacting proteins under physiological circumstances.

To circumvent the limitations and detect RBPs that bind to endogenous IncRNA transcripts in living cells, we developed a method termed CRISPR-Assisted RNA-Protein Interaction Detection (CARPID) (Figure 1). A nuclease activity free form of a compact Type VI-D RNA CRISPR single-effector system dCasRx was employed for specific IncRNA targeting ${ }^{7}$. This dCasRx effector protein retained the capability of processing guide RNA (gRNA) arrays to two or more component gRNAs without cleaving targeted RNA transcripts. Taking this advantage, we designed a guide RNA array composed of two gRNA sequences spaced by a 30-nt direct repeat (DR) to target two adjacent loci on the same IncRNA transcript. This theoretically allows augmented targeting specificity and thus reduced background noise. 
To identify the RBPs bound to the target IncRNA, we fused the dCasRx with the engineered biotin ligase BASU (Figure 2). To monitor and minimize the variation caused by heterogeneous expression of BASU enzyme among cells, we cloned the BASU-dCasRx in-frame with a self-cleaving T2A peptide and an eGFP CDNA. CARPID was carried out by co-expressing the BASU-dCasRx chimeric protein with gRNAs targeting specific regions of a IncRNA, inducing biotinylation, and subsequent enriching biotinylated proteins for mass spectrometry-mediated protein identification and quantification. Here we provide the protocol that we have optimized for CARPID.

\section{Reagents}

HEK293T cell line (some other cell lines of interest);

Appropriate cell culture media;

Molecular cloning reagents;

Biotin (Sigma \#B4639. Dissolve biotin with DMSO to the concentration of $100 \mathrm{mM}$. Can be stored at $-20^{\circ} \mathrm{C}$ for at least half a year.);

Dynabeads ${ }^{\circledR}$ MyOne ${ }^{\mathrm{TM}}$ Streptavidin T1 (ThermoFisher \#65601);

Tris-HCl (pH 7.5) (ThermoFisher \#15567027);

Tris-HCl (pH 8.0) (ThermoFisher \#15568025);

2-Mercaptoethanol (Sigma \#M3148);

Protease Inhibitor (Roche \#4693132001);

Pierce Protein Quantitation Assay (ThermoFisher \#23225);

4-20\% Bis-Tris gel (Bio-Rad \#4561095);

PVDF membrane (Millipore \#ISEQ00010);

Blocker $^{\text {TM }}$ BSA (10X) in TBS (ThermoFisher \#37520);

DTT (Sigma \#43815);

Ammonium bicarbonate (Alfa Aesar \#14249);

Sequencing Grade Trypsin (Pierce \#90058);

Acetonitrile (LiChrosolv $\left.{ }^{\circledR} \# 75-05-8\right)$;

C18 tips (ThermoFisher \#87782); 
Reverse phase C18 column (ThermoFisher \#164568);

Formic acid (Sigma \#5330020050);

lodoacetic acid (Pierce \#35603);

PEI (Polysciences, \#23966-1. Dissolve PEI with Milli-Q water to the concentration of $0.1 \%$. Can be stored at $-20^{\circ} \mathrm{C}$ for up to one year.);

Lysis buffer for Western Blot (50 mM Tris-HCl pH 7.4, $150 \mathrm{mM} \mathrm{NaCl}, 0.5 \%$ Triton X-100, 1 mM EDTA supplemented with fresh protease inhibitors);

Elution buffer for Western Blot (2x Laemmli Sample Buffer (Bio-Rad \#1610737), 8\% 2-Mercaptoethanol, 5 mM Biotin);

Elution buffer I for Mass Spectrometry (50 mM Tris-HCl pH 8.0, $2 \mathrm{M}$ urea, $10 \mu \mathrm{g} / \mathrm{ml}$ Sequencing Grade Trypsin, 1 mM DTT);

Elution buffer II for Mass Spectrometry (50 mM Tris-HCl pH 8.0, $2 \mathrm{M}$ urea, $5 \mathrm{mM}$ iodoacetamide).

\section{Equipment}

Standard molecular biology lab equipment;

DynaMag $^{\text {TM}}$-2 Magnet (ThermoFisher, \#12321D);

Easy-nLC 1200 system (Thermo Scientific);

Q Exactive HF mass spectrometry (Thermo Scientific);

XCalibur 4.0.27 (Thermo Scientific);

Proteome Discoverer 2.2 software (ThermoFisher).

\section{Procedure}

\section{Design and construct gRNA plasmids for targeting the IncRNA of interest.}

1.1 Design the paired gRNA sets with an in-house computational program (gp-designer). Typing in the gene symbol into the program, the full-length sequence of the IncRNA will be extracted for constructing (length=30nt, step $=1 \mathrm{nt})$ and filtering (GC\%=40\% 60\%) the sequence substrings. The uniquely aligned (human reference genome, hg19) and paired sequences (spacing, 16 20nt) are exported into a excel sheet with indicated RNAfold structure ratio (DotPercent, high value of "DotPercent" from RNAfold suggests a low chance of forming hairpin structure); 
1.2 Choose one or several sets of gRNAs targeting specific regions of IncRNA of interest and clone them into the pre-gRNA plasmid (Addgene \#109054) with a Gibson assembly approach (NEB, \#E2611S).

\section{Cell preparation and transfection.}

Before CARPID assay, evaluate the specificity of selected gRNA sets by co-expressing them with wild-type CasRx nuclease (Addgene \#109049) and determine the knockdown efficacy via quantitative RT-PCR (Figure 3).

2.1 Seed $4 \times 10^{6} \mathrm{HEK} 293 \mathrm{~T}$ cells in each $10-\mathrm{cm}$ plate one day before transfection. Cells are cultured in DMEM (Life Technologies) supplemented with 10\% inactivated FBS (Life Technologies) and 1\% penicillinstreptomycin (Life Technologies \#15140163) at $37^{\circ} \mathrm{C}$ with $5 \% \mathrm{CO}_{2}$;

2.2 Co-transfect $4 \mu \mathrm{g}$ of gRNA expressing plasmid and $4 \mu \mathrm{g}$ of BASU-dCasRx plasmid with $24 \mu \mathrm{g}$ of $0.1 \%$ PEl for each $10-\mathrm{cm}$ plate;

2.3 At 24 hours post-transfection, change the medium with fresh medium.

\section{Biotin labeling}

3.1 At 48 hours post-transfection, change the medium with fresh medium containing $200 \mu \mathrm{M}$ biotin and incubate for 15 mins at $37^{\circ} \mathrm{C}$ with $5 \% \mathrm{CO}_{2}$;

3.2 Replace the medium with $10 \mathrm{ml}$ ice-cold PBS to quench the reaction;

3.3 Wash cells with $10 \mathrm{ml}$ ice-cold PBS at least three times to remove the remaining biotin.

\section{Cell lysis}

4.1 Add $1 \mathrm{ml}$ ice-cold PBS to the plate and scrape the cells off, transfer them to a $15 \mathrm{ml}$ tube and pellet the cells by centrifugation at $1,200 \mathrm{rpm}$ for 5 mins;

4.2 Completely remove the PBS and resuspend the cell pellet in $1 \mathrm{ml}$ cell lysis buffer;

4.3 After end-over-end rotating in cold room for $10 \mathrm{mins}$, spin the lysate at $15,000 \mathrm{rpm}$ for $10 \mathrm{~min}$ at $4^{\circ} \mathrm{C}$ to remove the cell debris;

4.4 Transfer the supernatant to a new centrifuge tube and measure the protein concentration with Pierce Protein Quantitation Assay; 
4.5 Adjust the volume the lysates to equalize the protein concentration;

4.6 Set aside $50 \mu$ of lysate as Input, and use the remaining lysates for the following immunoprecipitation.

\section{Streptavidin immunoprecipitation}

5.1 Add $30 \mu$ of MyOne T1 streptavidin beads to the lysate and incubate in cold room for 2 hours with end-over-end rotation;

5.2 Precipitate the streptavidin beads with DynaMag ${ }^{\mathrm{TM}}-2$ Magnet stand and remove the supernatant completely;

5.3 Resuspend the beads with $1 \mathrm{ml}$ ice-cold lysis buffer, and perform end-over-end rotation for 5 mins;

5.4 Repeat Step 5.2 and 5.3 twice and place the tube on the DynaMag ${ }^{\mathrm{TM}}-2$ Magnet stand.

\section{CARPID-Western Blot (CARPID-WB)}

6.1 Remove the supernatant completely and add $30 \mu$ elution buffer to heat elute the biotinylated proteins from the streptavidin beads at $95^{\circ} \mathrm{C}$ for $10 \mathrm{~min}$ and label it as IP;

6.2 Load the Input and IP samples to $4-20 \%$ Bis-Tris gel and transfer proteins to a PVDF membrane;

6.3 Block the membrane with BSA blocker for 1 hour at room temperature (RT) and subsequently incubate the blocked membrane with indicated primary antibodies in cold room overnight;

6.4 After primary antibody incubation, wash the membrane with $1 \times$ TBST for three times at RT;

6.5 Incubate the membrane with the corresponding secondary antibodies for $1 \mathrm{~h}$ at RT;

6.6 Wash the membrane with $1 \times$ TBST for three times and apply appropriate amount of ECL substrate to the washed membrane at RT;

6.7 Detect the signal with the Bio-Rad ChemiDoc Imaging System;

6.8 For signal quantification, use ImageJ software (version 1.8.0_172) to quantify and normalize the WB signals (Figure 4).

\section{CARPID-Mass Spectrometry (CARPID-MS)}


7.1 For CARPID-MS assay (follow step 5.4), wash the streptavidin beads further with $1 \mathrm{ml}$ of $50 \mathrm{mM}$ ammonium bicarbonate $(\mathrm{pH} 8.0)$ at $4^{\circ} \mathrm{C}$ for three times;

7.2 Resuspend the beads in $50 \mu \mathrm{l}$ Elution buffer I and incubate at $30^{\circ} \mathrm{C}$, mixing at $400 \mathrm{rpm}$ for $60 \mathrm{mins}$ and collect the supernatant to a fresh tube;

7.3 Resuspend the beads with $25 \mu$ l Elution buffer II twice and combine all three batches of elutes. Protect samples from light during this procedure;

7.4 Add additional $0.25 \mu \mathrm{g}$ trypsin into the combined elutes and incubate at $37^{\circ} \mathrm{C}$ overnight;

7.5 Add $10 \%$ formic acid at a ratio of 1:25 (v/v) to stop the digestion;

7.6 Desalt the digested samples with C18 tips following manufacturer's instruction and reconstitute in 20 $\mu \mathrm{l}$ of $0.1 \%$ formic acid;

7.7 Perform the LC-MS/MS analysis using an Easy-nLC 1200 system coupled to a Q Exactive HF mass spectrometry. Inject $6 \mu$ l of samples to a reverse phase C18 column $(75 \mu \mathrm{m}$ i.d. $\times 15 \mathrm{~cm}, 3 \mu \mathrm{m}$ particle size, Thermo Scientific \#164568) at a flow rate of $250 \mathrm{nl} / \mathrm{min}$. Mobile phase A $(0.1 \%$ formic acid in ultrapure water) with an eluting buffer as mobile phase $B(0.1 \%$ formic acid in $80 \%$ acetonitrile) are used together to establish a linear 50 -min gradient of $7-25 \%$ mobile phase $B$;

7.8 Peptides are ionized by electrospray at $2.3 \mathrm{kV}$. The mass spectrometer is operated in positive ion mode acquiring at a resolution of 120,000 , with a full MS spectrum $(\mathrm{m} / \mathrm{z}=350-1800)$ using an automatic gain control (AGC) target of $3 \times 10^{6}$. The top 12 most intense ions are selected for higher-energy collisional dissociation (HCD) fragmentation (normalized collision energy 27) and MS/MS spectra are generated with an AGC target of $1 \times 10^{5}$ at a resolution of 30,000 . The dynamic exclusion time is set to $30 \mathrm{~s}$.

\section{LC-MS/MS Data Processing and Analysis}

8.1 Analyze all the raw files created by XCalibur (version 4.0.27) software together with the Proteome Discoverer version 2.2 software, against the UniProt human protein database in Sequest HT node.

8.2 Set the precursor and fragment mass tolerances to $10 \mathrm{ppm}$ and $0.02 \mathrm{Da}$, respectively. The maximum of two missed cleavage sites of trypsin is allowed;

8.3 Set carbamidomethylation (C) as static modification and set oxidation (M) and acetyl (protein $\mathrm{N}$ terminal) as variable modifications. False discovery rate (FDR) of peptide spectrum matches (PSMs) and peptide identification are determined using the Percolator algorithm at $1 \%$ based on q-value;

8.4 For label-free quantification (LFQ), use the Minora Feature Detector node in the processing workflow, and use the Precursor lons Quantifier node and the Feature Mapper node in the consensus workflow; 
8.5 Apply enrichment analysis for proteins with more than one peptide detected. Human keratins are included. Normalize the LFQ abundances across the pulldowns and logarithmized. Impute the missing values with values representing the detection limit of the mass spectrometer. Apply the rank product test to determine proteins statistically enriched in the gRNA transfected samples compared to the pre-gRNA controls. Proteins with an adjusted $p$-value of $\leq 0.05$ and $\geq 2$-fold change of abundance are considered significant RBPs of the IncRNA of interest (Figure 5).

\section{Troubleshooting}

\section{Time Taken}

\section{Anticipated Results}

\section{References}

1 Consortium, E. P. An integrated encyclopedia of DNA elements in the human genome. Nature 489, 57-74, doi:10.1038/nature11247 (2012).

2 Djebali, S. et al. Landscape of transcription in human cells. Nature 489, 101-108, doi:10.1038/nature11233 (2012).

3 Chu, C. et al. Systematic discovery of Xist RNA binding proteins. Cell 161, 404-416, doi:10.1016/j.cell.2015.03.025 (2015).

4 Castello, A. et al. System-wide identification of RNA-binding proteins by interactome capture. Nat Protoc 8, 491-500, doi:10.1038/nprot.2013.020 (2013).

5 Selth, L. A., Gilbert, C. \& Svejstrup, J. Q. RNA immunoprecipitation to determine RNA-protein associations in vivo. Cold Spring Harb Protoc 2009, pdb prot5234, doi:10.1101/pdb.prot5234 (2009).

6 Licatalosi, D. D. et al. HITS-CLIP yields genome-wide insights into brain alternative RNA processing. Nature 456, 464-469, doi:10.1038/nature07488 (2008).

7 Konermann, S. et al. Transcriptome Engineering with RNA-Targeting Type VI-D CRISPR Effectors. Cell 173, 665-676 e614, doi:10.1016/j.cell.2018.02.033 (2018).

\section{Acknowledgements}

We are grateful to Prof. Bing Ren (UC San Diego) and Dr. Danny C.Y. Leung (HKUST) for insightfulcomments during manuscript preparation. This work was supported by the City University of Hong Kong (7200595, 7005314, 9667188 and 9610424), the National Natural Science Foundation of China (81873642 and 31900443), Research Grants Council of Hong Kong $(21100615,11102118$, 
11101919, C7007-17GF to K.M.C., and 21101917, 11103318 to L.Z.), the Shenzhen Science and Technology Fund Program (JCYJ20170818104203065, JCYJ20180307124019360 to K.M.C., JCYJ20170413141047772, JCYJ20180507181659781 to L.Z.); Opening Foundation of Key Laboratory of Resource Biology and Biotechnology in Western China (Northwest University), the Chinese Ministry of Education; and the Hong Kong Epigenomics Project of the EpiHK consortium (Lo Ka Chung Charitable Foundation) (J.Y. and K.M.C.).

\section{Figures}

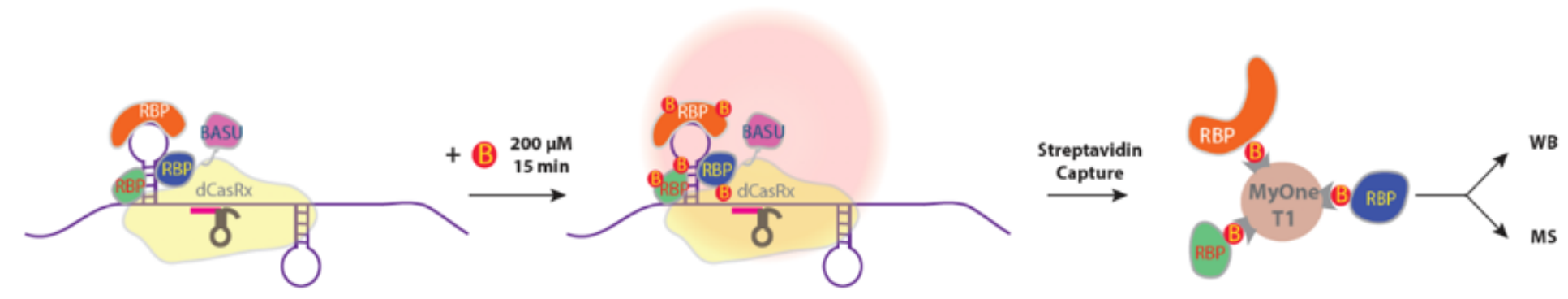

\section{Figure 1}

Figure 1: Scheme of the CARPID workflow. A IncRNA of interest is targeted by a set of gRNAs. The catalytically dead RNA nuclease CRISPR/CasRx (dCasRx) fused with the engineered biotin ligase (BASU) is recruited to the specific locus. Following biotin treatment, the adjacent RNA binding proteins (RBPs) would be biotinylated by BASU. The labelling radius of the BASU-dCasRx is indicated by the red shading. Biotinylated proteins are enriched by streptavidin beads (MyOne T1) for subsequent mass spectrometric (MS) identification and western blot (WB) analysis.

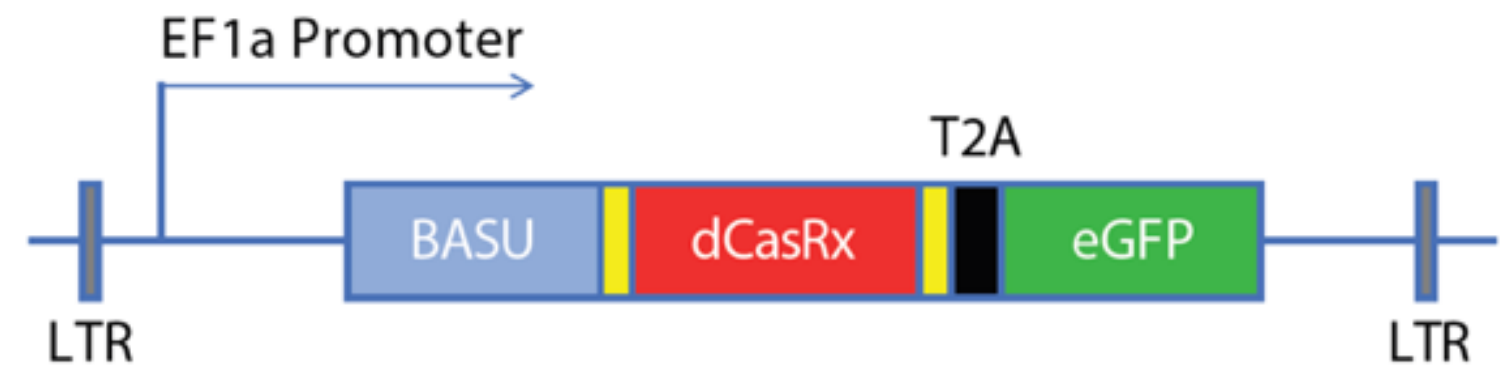

\section{Figure 2}

Figure 2: Scheme of BASU-dCasRx construct. BASU was subcloned from the BASU RaPID plasmid (Addgene \#107250) and cloned in-frame into the EF1a-dCasRx-2A-EGFP plasmid (Addgene \#109050). LTR, long terminal repeat; T2A, self-cleaving peptide; eGFP, enhanced GFP. 


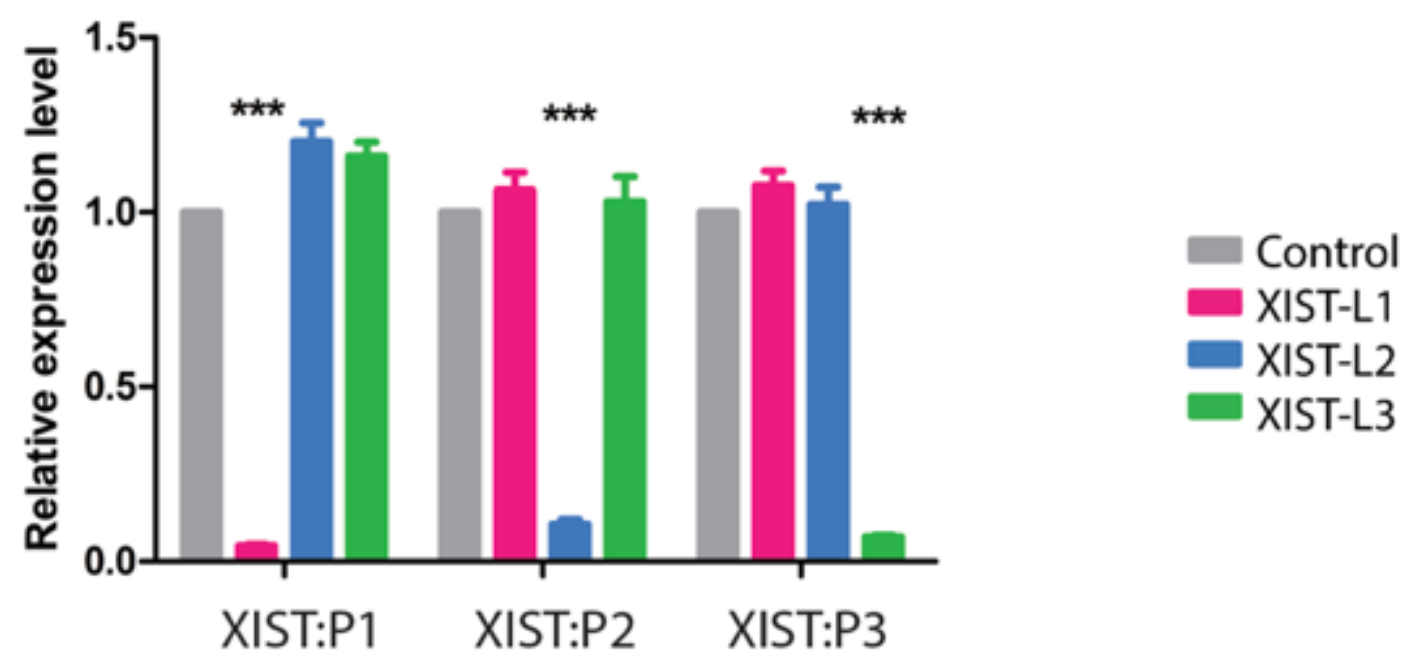

Figure 3

Figure 3: Reverse transcription qPCR shows the specificity of the CRISPR/CasRx system in XIST. HEK293T cells were co-transfected with CasRx and individual gRNA (empty vector control, XIST-L1, XISTL2 and XIST-L3), respectively. Total RNA was extracted from treated cells followed by reverse transcription and qPCR assay to quantify the level of specific loci of XIST. GADPH was used to normalize the level of XIST. Note that active CasRx together with a set of gRNAs specifically decreased the level of RNA transcript at its targeting locus. Data are represented as mean $\pm S D, n=3, * \star \star p<0.001$, unpaired Student's t-test. 


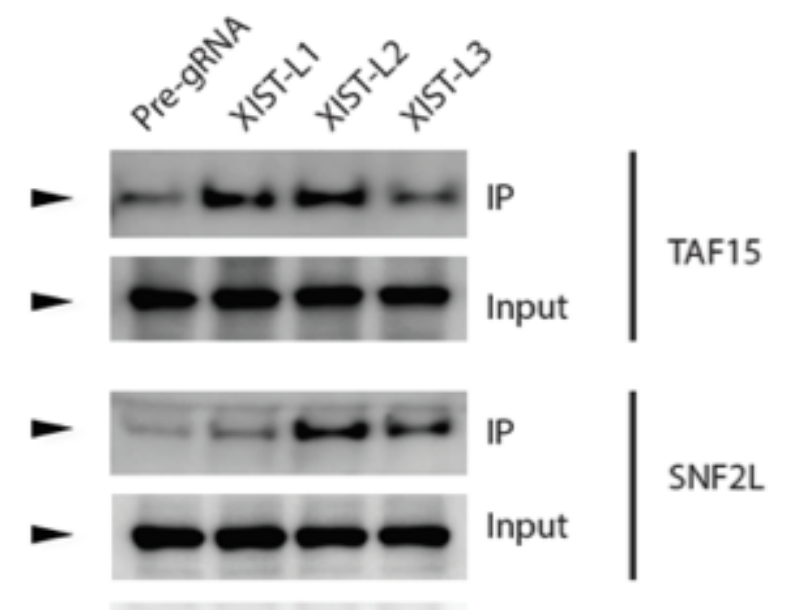

Biotin

\section{Figure 4}

Figure 4: CARPID followed by Western blot using antibody against human TAF15 and SNF2L.

Precipitations of biotinylated proteins were conducted by applying streptavidin beads to the lysates of HEK293T cells transfected with pre-gRNA (Control), gRNA set specific for locus 1 (XIST-L1), locus 2 (XISTL2), and locus 3 (XIST-L3) together with BASU-dCasRx, respectively. Three biological replicative experiments were carried out, and a representative result is shown. ImageJ software (version 1.8.0_172) was employed to quantify the WB signals and normalized targets IP signals with biotin-BASU-dCasRx (biotin). 


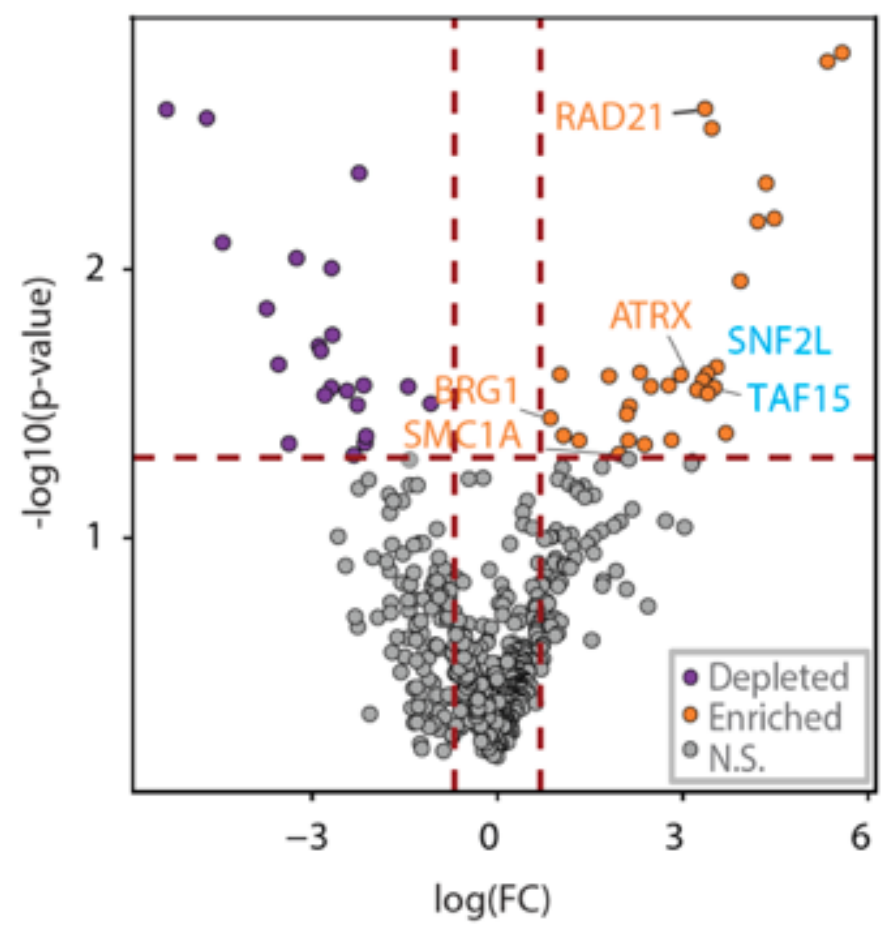

\section{Figure 5}

Figure 5: Identification of XIST-associated proteome with CARPID. The volcano plot shows the enrichment of XIST-associated proteins in HEK293T cells. The $x$-axis indicates the log2 fold change of protein levels in CARPID combining all three sets of XIST gRNAs relative to control. The y-axis shows the negative logarithm transformed $p$ value (the non-parametric rank product test). Significantly enriched proteins are labelled as orange dots. Proteins previously validated to interact with XIST and identified by CARPID are labelled in orange typeface. SNF2L and TAF15, two novel XIST-associated proteins, are indicated by blue typeface. 